\title{
Shipborne Missile Weapon Assignment Study
}

\author{
Xie Chunsi ${ }^{1, a}$ \\ ${ }^{1}$ Missile and Cannon Department, 667\#, Jiefang Road, Dalian City, China \\ axiechunsi@163.com
}

Keywords: Shipborne; Missile Weapon; Algorithm

Abstract. Aiming at the issue of constrained optimization problem of assignment for shipborne missile weapon, hybrid genetic algorithm has been proposed in this paper, which can get global optimization results of the assignment, and provide decision support for the assignment.

\section{Introduction}

Suppose that shipborne missile weapon uses semi-active radar homing, the assignment for the system is to assign the illumination radars of the system to the arriving targets. The shipborne missile fire effect is affected by the solution quality of the assignment scheme.

The assignment is a typical integer programming problem and belongs to 0-1 Programming problem. Genetic algorithm a searching arithmetic of global optimization (Mitchell Melanie, 1999), based on principles inspired by natural genetics "survival of the fittest, excellent win and inferior discard", can be used to solve the assignment. Previous work used standard genetic algorithm to solve the optimization problem of the assignment for the system (Youtang Tong, 2009). However, standard genetic algorithm is of insufficient local optimization, in contrast to hill climbing (Kenekayoro Patrick, 2012), and the two methods can be combined with each other to form hybrid genetic algorithm that can be proposed to get global optimization results.

\section{Assignment Model}

It is supposed that there are $\mathrm{N}$ arriving targets, and the system has $\mathrm{M}$ illumination radars, $\mathrm{M} \geq 1$. Ensuring maximal targets to meet the illumination radar irradiation conditions is considered as optimization objective (Yang Zhang, 2012), and then supposing that target number which meet the illumination radar irradiation conditions is defined by $\mathrm{f}$ whose maximization is the key of the assignment problem.

$$
\begin{gathered}
\max f=\sum_{i=1}^{M} \sum_{j=1}^{N} R_{j} X_{i j} \\
g_{j}=\sum_{i=1}^{M} X_{i j}-b_{j} \leq 0 \\
g_{N+i}=\sum_{j=1}^{N} X_{i j}-1 \leq 0
\end{gathered}
$$

where $R_{j}$ is the $j$-th target threat value, $X_{i j}$ is a Boolean value used to determine whether the $i$-th illumination radar is assigned to the $\mathrm{j}$-th target. If the $\mathrm{i}$-th illumination radar is assigned to the $\mathrm{j}$-th target, then $X_{i j}=1$, otherwise $X_{i j}=0$. Eq. (2) represents that the number of illumination radar assigned to the $\mathrm{j}$-th target doesn't exceed $b_{\mathrm{j}}$. Eq. (3) represents that the $\mathrm{i}$-th illumination radar is assigned to at most one target.

The constraints of the assignment model mainly include commander's decision and the illumination radar coverage.

\section{Decision Constraint}

Because of the complex campaign circumstances, the achieved data is impossible to be complete, determinate and reliable. Commander's decision is necessary, namely, the commander should have 
definite interference on the assignment scheme. If the $\mathrm{j}$-th target is of very importance, the $\mathrm{R}_{\mathrm{j}}$ value of the $\mathrm{j}$-th target given by the commander's decision can have significant impact on the assignment scheme.

\section{Illumination Radar Coverage Constraint}

For single illumination radar (let A radar), only when the position of a single target is within the A radar coverage, can the target be illuminated by the A radar.

Let the time for the first target to reach the long-range limit of the A radar coverage be $T_{Y}(A, 1)$, and the time for the first target to stay within the $A$ radar coverage be $T_{D}(A, 1)$, and the time for the first target to be initially illuminated by the A radar be $T_{L}(A, 1)$. The first target can be effectively illuminated by the A radar by satisfying Eq. (4).

$$
\mathrm{T}_{\mathrm{Y}}(\mathrm{A}, 1) \leq \mathrm{T}_{\mathrm{L}}(\mathrm{A}, 1) \leq \mathrm{T}_{\mathrm{Y}}(\mathrm{A}, 1)+\mathrm{T}_{\mathrm{D}}(\mathrm{A}, 1)
$$

After the completion of the illumination task of the first target, the second target is illuminated by the A radar which requires a transfer time to initialize work. Let the minimum transfer time for the A radar be $\mathrm{T}_{\mathrm{Z}}$, and then the second target can be effectively illuminated by the A radar by satisfying Eq. (5) and Eq. (6).

$$
\begin{gathered}
\mathrm{T}_{\mathrm{Y}}(\mathrm{A}, 2) \leq \mathrm{T}_{\mathrm{L}}(\mathrm{A}, 2) \leq \mathrm{T}_{\mathrm{Y}}(\mathrm{A}, 2)+\mathrm{T}_{\mathrm{D}}(\mathrm{A}, 2) \\
\mathrm{T}_{\mathrm{Z}} \leq \mathrm{T}_{\mathrm{L}}(\mathrm{A}, 2)-\mathrm{T}_{\mathrm{L}}(\mathrm{A}, 1)
\end{gathered}
$$

where $T_{Y}(A, 2)$ is the time for the second target to reach the long-range limit of the A radar coverage, $T_{D}(A, 2)$ is the time for the second target to stay within the A radar coverage, $T_{L}(A, 2)$ is the time for the second target to be initially illuminated by the A radar.

\section{Genetic Algorithm Based on Adaptive Penalty Function}

\section{Fitness function design}

The assignment model is a constrained optimization problem which can't be directly solved by genetic algorithm. By introducing the penalty function, the constrained optimization problem is converted into an unconstrained optimization problem which can be solved by genetic algorithm.

This paper uses adaptive penalty function (Qiongsun Liu etc, 2006), and the penalty factor is a function of the independent variable $\mathrm{X}_{\mathrm{ij}}$. Meanwhile, in order to accelerate the convergence speed, this paper draws a "multi-level punishment" thinking which gives bigger constraint violation severer punishment, and smaller constraint violation lesser punishment. The fitness function can be expressed as in Eq. (7).

$$
\begin{gathered}
\text { eval }=f+C \cdot p \\
p=\sum_{k=1}^{M+N} Q_{k} \cdot \max \left\{0, g_{k}\right\} \\
Q_{k}=\frac{\max \left\{0, g_{k}\right\}}{\sum_{l=1}^{M+N} \max \left\{0, g_{l}\right\}} \\
C=1+\frac{|f|}{1+p}
\end{gathered}
$$

where $\mathrm{p}$ is penalty function, $\mathrm{C}$ is the penalty function coefficient.

\section{Genetic Algorithm Design}

\section{Standard Genetic Algorithm Design}

Binary encoding. Binary coding handles each chromosome as cord of bits, either 1 or 0 . The length of individual chromosome is $M \times N$. The chromosome can be expressed as $A_{1}^{k_{h}} A_{2}^{k_{h}} \mathrm{~L} A_{M}^{k_{h}}, A_{i}^{k_{h}}=$ 
$\left(X_{i 1}^{k_{h}} X_{i 2}^{k_{h}} \mathrm{~L} X_{i N}^{k_{h}}\right) . X_{i j}^{k_{h}}$ is string $k_{h}$ of the h-th generation, which represents the $\mathrm{i}$-th illumination radar assigned to the $\mathrm{j}$-th target.

Initial population. Standard genetic algorithm generates initial population by the random method, and each chromosome of initial population might or might not satisfy the constraints defined in Eq. (2) and Eq. (3). The following method can automatically generate initial population which satisfies the constraints. The steps are as follows: first of all, set ' 0 ' to all genes in all chromosomes in the population, then each chromosome is transferred to a matrix ranked $M \times N$. In each column, one or two genes are randomly set ' 1 ' so that the matrix satisfies the constraint defined in Eq. (3). Next, in each row, several genes are randomly set ' 1 ' so that the matrix satisfies the constraint defined in Eq. (2), and the third step is stop.

Fitness evaluation. Each individual represents a assignment scheme, which should meet the illumination radar coverage constraint. This paper implements fitness evaluation based on Eq. (7). The A radar coverage constraint test method is as follows. Based on the sequence of the arriving targets into the A radar coverage, the method determines the A radar illumination sequence for the arriving targets. Which target arrives first, the target is on the first illumination. When two or more targets enter the A radar coverage simultaneously, the priority is given to the target of the highest threat value for the A radar. For the primary illumination of the A radar, it can be determined whether the first target is effectively illuminated based on Eq. (4). If not so, the corresponding gene in the chromosome for the A radar illuminating the first target is set to be ' 0 '. When the A radar still has the task of illuminating other targets, it selects another target as the first target based on Eq. (4), and sets the corresponding gene in the chromosome for the A radar illuminating the target. If so, it should determine whether the A radar still has the task of illuminating the second target. If so, it should determine whether the second target can be effectively illuminated by the A radar based on Eq. (5) and Eq. (6), If not, the corresponding gene in the chromosome for the A radar illuminating the second target is set to be ' 0 ', when the the A radar still has the task of illuminating other targets, it selects another target as the second target based on the above procedure. If so, it should determine whether the A radar still has the task of illuminating the third target. The above process is repeated until the assignment scheme satisfies the A radar coverage constraint.

Selection operation. The selection operation is roulette wheel selection in the standard genetic algorithm. This paper adopts the selection strategy of associative proportional selection and distillate model, which selects the individual of maximal fitness value in the $\mathrm{H}$ individuals of each generation directly to be the individual of the next generation, while the other individuals of the next generation are engendered by the remaining $\mathrm{H}-1$ individuals of the former generation through the roulette wheel selection method. This selection strategy, on the one hand, can guarantee that the most excellent individual survives to the next generation, on the other hand, it can have the avoidance that the chance to select the next generation is in great disparity because of each individual's different fitness value, which guarantees the diversity of population to a certain extent.

Crossover operation. Selecting two individuals randomly in the population as parent individuals. Suppose that they are chromosome $k_{h_{1}}$ (which is defined by $A_{1}^{k_{h_{1}}} A_{2}^{k_{h_{1}}} \mathrm{~L} A_{M}^{k_{h_{1}}}$ ) and chromosome $k_{h_{2}}$ (which is defined by $A_{1}^{k_{h_{2}}} A_{2}^{k_{h_{2}}} \mathrm{~L} A_{M}^{k_{h_{2}}}$ ) of the h-th generation. Interchanging $A_{1}^{k_{h_{1}}}$ and $A_{M}^{k_{h_{2}}}$, interchanging $A_{2}^{k_{h_{1}}}$ and $A_{M-1}^{k_{h_{2}}}$, interchanging $A_{3}^{k_{h_{1}}}$ and $A_{M-2}^{k_{h_{2}}}$, and so on, eventually two new individuals are generated.

Mutation operation. For the chromosome $k_{h}$ of the h-th generation, $A_{d 1}^{k_{h}}$ and $A_{d 2}^{k_{h}}$ are randomly selected, then interchanged them to form two new chromosomes, $A_{e 1}^{k_{h}}$ and $A_{e 2}^{k_{h}} . \mathrm{d} 1, \mathrm{~d} 2$, e1, $\mathrm{e} 2=1,2, \ldots, \mathrm{M}$, the value of $\mathrm{d} 1, \mathrm{~d} 2$, e1, and e 2 are different. Then the fitness values of the two newly created individuals are compared with the two parent individuals, if the same values, invalid mutation operation is considered to remove the two newly created individuals, and mutation operation is performed according to the above procedure. Otherwise, valid mutation operation is considered, and it substitutes the two newly created individuals for the two parent individuals. 
Algorithm termination condition. Let $\varepsilon$ be a small positive number, when the absolute difference between the fitness function values of two neighboring generations is smaller than $\varepsilon$, the standard genetic algorithm terminates.

\section{Hill Climbing Design}

Bit hill climbing is adopted because binary coding handles each chromosome, whose bit variation style is a variation of the order from left to right (Xiaozhen Yan etc, 2007). Let the single best individual from the above standard genetic algorithm be $k_{y}$ with the length $M \times N$. The search process of the bit hill climbing is as follows.

Step one. Suppose the mutation of the $\mathrm{d}$-th bit. The initial value of $\mathrm{d}$ is 1 .

Step two. The mutation of the d-th bit of $k_{y}$ inverts the bit value from '0' to '1', or '1' to '0', to obtain new individual (defined by $k_{x}$ ).

Step three. Based on the above fitness evaluation method, the fitness values of $k_{x}$ (defined by eval ${ }_{x}$ ), and $k_{y}$ (defined by eval ) can be calculated.

Step four. If $e v a l_{x}>$ eval,$k_{y}$ is replaced by $k_{x}$. Otherwise, it is back to step two, and $\mathrm{d}=\mathrm{d}+1$. So it is constantly repeated until $\mathrm{d}=M \times N$, and the best individual can be obtained.

\section{Conclusions}

The proposed hybrid genetic algorithm based on adaptive penalty function can solve constrained optimization problem of assignment for shipborne missile weapon. The constrained optimization problem is converted into the unconstrained one through the introduction of adaptive penalty function. Afterwords, hybrid genetic algorithm is proposed by combining standard genetic algorithm with hill climbing to solve the unconstrained optimization problem, and to get global optimization results of the assignment, which helps the assignment decision for the system.

\section{References}

[1] Kenekayoro Patrick. "Comparison of simulated annealing and hill climbing in the course timetabling problem." African Journal of Mathematics and Computer Science Research, Vol. 5 (11): 176-178.(2012)

[2] Mitchell Melanie. An Introduction to Genetic Algorithms. Cambridge: MIT Press.(1999)

[3] M. Srinivas and L.M. Patnaik. "Adaptive probabilities of crossover and mutation in genetic algorithms.” IEEE Trans. Systems, Man Cybernet. Vol. 24 (4): 656-667.(1994)

[4] Qiongsun Liu, Shenghua Zhou. "Hybrid Genetic Algorithm Based on Novel Adaptive Penalty Function." Journal of Chongqing University, Vol. 29 (6): 78-81.(2006)

[5] Xiaozhen Yan, Hong Xie. Yao Liu. "Mixed multiuser detection based on genetic algorithm and bit hill climbing algorithm.” Applied Science and Technology, Vol. 34 (8): 29-33.(2007) 